\title{
Análisis de las Actitudes de la Educación Física en la E.S.O Attitudinalanalysis of Physical Education in Compulsory Secondary Education (E.S.O.)
}

\author{
* José Luis Chinchilla Minguet, **Manuel Francisco Escribano Castillo, *Oscar Romero Ramos, *Iván López Fernández \\ *Universidad de Málaga, **I.E.S. Blas Infante de Córdoba
}

\begin{abstract}
Resumen: Objetivo del trabajo: crear un programa de evaluación de las actitudes en Educación Física para la E.S.O. 1. Diseñar un modelo de evaluación de las Actitudes para Educación Física. 2. Crear instrumentos de recogida, análisis e interpretación de datos. 3. Validez de los objetivos. 4. Verificar la hipótesis. Hipótesis: «cuanta más información se disponga sobre un alumno, disminuyen las posibilidades de errar en su calificación». Metodología: se ha seguido un Diseño Longitudinal de Panel. Dos grupos de trabajo, $4^{\circ}\left\langle\mathrm{F} »\right.$ y, $4^{\circ}$ «G» de E.S.O., I. E. S. de Málaga «Universidad Laboral», curso 06/07. $4^{\circ} \mathrm{F}$ evaluado con el modelo tradicional de evaluación, $4^{\circ} \mathrm{G}$ con el experimental. En los dos grupos se recogieron variables actitudinales: las variables independientes se aplicaron al grupo experimental, y las dependientes al grupo control. Los resultados, se analizaron, para ver si la variable independiente, era la causante de la mejora en los resultados. Se ha empleado la observación directa, la recogida de datos, utilizando instrumentos: cuadernos de registros de $4^{\circ}$ de E.S.O., hojas personales de actitudes por ámbitos de actuación, diario de observación del alumno, y grupo, y libro de texto para $4^{\circ}$ de E.S.O.Conclusión: todo proceso de evaluación es complejo y en Educación Física más, por el volumen, la variedad, diversidad y la procedencia de la información, por diseñar instrumentos de recogida de datos, por individualizar el proceso, por estar el alumnado en movimiento.Los resultados afirman que la evaluación es eficaz, cuanta más información se tenga.
\end{abstract}

Palabras claves: Actitudes, Evaluación, Constructivista, Conductas.

\begin{abstract}
Objective of the work: to create a program of evaluation of the attitudes in Physical education for the E.S.O. 1. To design a model of evaluation of the Attitudes for Physical education. 2. To create instruments of collection, analysis and data processing. 3. Validity of the objectives. 4. To verify the hypothesis. The hypothesis: «whatever more information is arranged on a student, diminish the possibilities of being mistaken in its qualification». Methodology: diseño Longitudinal of Panel has been followed. Two work groups, $4^{\circ}\left\langle\mathrm{F} »\right.$ and, $4^{\circ}$ «G» of E.S.O., I. E. S. of Malaga «Labor University», course $06 / 07.4^{\circ} \mathrm{F}$ evaluated with the traditional model of evaluation, $4^{\circ} \mathrm{G}$ with the experimental one. In both groups actitudinales variables took shelter: the independent variables were applied to the experimental group, and the employees to the group control. The results, were analyzed, to see if the independent variable, were the cause of the improvement in the results. The direct observation, the collection of data has been used, using instruments: notebooks of registries of $4^{\circ}$ of E.S.O., personal leaves of attitudes by scopes of performance, record book of the student, and group, and text book for $4^{\circ}$ of E.S.O. Conclusion: all process of evaluation is complex more and in Physical education, by the volume, the variety, diversity and the origin of the information, to design instruments of collection of data, to individualize the process, to be the pupils in movement. The results affirm that the evaluation is effective, to whatever more information is had.
\end{abstract}

Key words: Attitudes, Evaluation, Constructivist, Behaviour.

\section{Introducción}

El fundamento esencial de la investigación ha sido diseñar un programa de evaluación de las actitudes en Educación Física para la Educación Secundaria Obligatoria, permitiendo realizar, un análisis y una profunda reflexión sobre este tema.

No se ha pretendido establecer un referente general y definitivo sobre la materia en cuestión, puesto que por definición, un trabajo de investigación, no limita, sino todo lo contrario, abre nuevas líneas de investigación y genera otras alternativas.

El trabajo se ha orientado hacia la práctica educativa de laEducación Física y se ha pretendido contribuir a que ésta se organice, de tal manera, que se pueda extender los resultados a otros sujetos, y a otros contextos educativos.

\section{Objetivos}

Cuatro han sido los objetivos que se han perseguido:

1. Diseñar un modelo de evaluación de las Actitudes para la Educación Física en Educación Secundaria Obligatoria, que facilite la tarea del profesor en la evaluación de las actitudes. (Sebastiani y Blázquez, 1998) en el trabajo titulado «Innovaciones y Desafios en la Evaluación de laEducación Física», nos ofrecen un enfoque más preciso e la evaluación cuando señalan que a pesar de haber estado asociada tradicionalmente a la calificación y a la atribución de una nota, el concepto de evaluación reviste actualmente una idea y un significado totalmente

Fecha recepción: 01-10-07 - Fecha envío revisores: 08-10-07 - Fecha de aceptación: 30-06-08 Correspondencia: José Luís Chichilla Minguet

Juan Sebastián El Cano, 154

29004 Málaga

E-mail: jlchinchilla@uma.es diferente. La evaluación es concebida en estos momentos, como la reflexión crítica sobre los componentes y los intercambios en cualquier proceso, con el fin de determinar cuáles están siendo, o han sido, sus resultados y poder tomar las decisiones más adecuadas para la positiva consecución de los objetivos perseguidos).

2. Diseñar instrumentos de recogida y almacenamiento de información, para su posterior análisis e interpretación, como manera de interpretar el proceso de evaluación (Stenhouse, 1984 «para evaluar hay que comprender»).

3. Se ha comprobado la validezy la fiabilidad de los dos objetivos antes mencionados.

4. Verificar con las conclusiones la verosimilitud de la hipótesis.

La hipótesis ha sido, «que cuanta más información relevante tengamos sobre el un alumno/a, las probabilidades de equivocarse en su calificación disminuyen proporcionalmente a la información manejada».

Se ha seguido una metodología, con intención educativa. Se ha deseado que el hecho educativo constituya el eje fundamental de la investigación y que dé sentido a la pedagógica. Se trata de diseñar estrategias argumentadas en un Paradigma Constructivista, (Abbot, 1999, «proceso subjetivo por el que cada persona va modificando constantemente a la luz de sus experiencias») para mejorar la práctica de la docencia en la Evaluación de las Actitudes en la Educación Física.

\section{Diseño de la Investigación.}

Se ha seguido un Diseño Longitudinal de Panel. Se han utilizado dos grupos de trabajo, $4^{\circ}\left\langle\right.$ F $» y, 4^{\circ}$ «G» de Educación Secundaria Obligatoria, del Instituto de Enseñanza Secundaria de Málaga «Universidad Laboral». El primer grupo ha sido sometido a un modelo tradicional de evaluación, y el segundo al modelo experimental. 


\begin{tabular}{|l|}
\hline \multicolumn{1}{|c|}{ VARIABLES DEPENDIENTES } \\
\hline \multicolumn{1}{|c|}{ EVALUACIÓN TRADICIONAL } \\
\hline \multicolumn{1}{|c|}{ GRUPO CONTROL } \\
\hline 1.- Examen teórico. \\
\hline 2.- Examen práctico. \\
\hline 3.- Diario de clase. \\
\hline 4.- Asistencia: \\
-F: no asiste a clase y no presenta justificante. \\
-FJ: no asiste a clase pero presenta justificante al reincorporarse. \\
-A: asiste a clase pero no realiza la práctica y no presenta justificante. \\
-AJ: asiste a clase, no realiza la práctica pero presenta justificante. \\
-ATJ: asiste a clase, colabora con el profesor y presenta el justificante. \\
-R: llega tarde de forma injustificada. \\
-V: no viene a clase de Educación Física con la indumentaria deportiva adecuada.
\end{tabular}

Muy positiva: supone el aumento de la nota media de la evaluación hasta en un punto.

Positiva: es la que se espera de todo alumno.

Negativa: supone la reducción de la nota media de la evaluación hasta en un punto.

Muy negativa: Supone el suspenso en esta asignatura.

\begin{tabular}{|l|}
\hline 6.- Grupos de trabajo. \\
\hline 7.- Trabajos. \\
\hline 8.- Monitores y árbitros. \\
\hline 9.- Participación en Actividades Deportivas Extraescolares. \\
\hline 10.- Pruebas físicas. \\
\hline
\end{tabular}

5.- Actitud:

5. Obtención de los datos, e instrumentos de recogida

En la obtención de datos se ha empleado la observación directa, y la recogida sistemática de información, utilizando instrumentos diseñados a tal fin: el Cuaderno de Registros de $4^{\circ}$ de E.S.O., las Hojas Personales de Registros de Actitudes por Ámbitos de Actuación, el Diario de Observación Personal de Clase, el Diario de Observación del Grupo Clase, y el libro de texto.

Se planteó la cuestión de ¡cuándo evaluar? Para lo que se diferenció, las fases de evaluación que marca la Ley, de los períodos que es conveniente poner en funcionamiento los procesos de evaluación. Los primeros están determinados en el calendario escolar, los segundos según establezcan las circunstancias.

Se creyó necesaria una evaluación inicial para determinar los conocimientos previos de los alumnos/as. Los resultados de la misma, se contrastaron con los resultados de la evaluación continua y de la final, y se observaron los cambios que se habían producido.

A los dos grupos se le han aplicado pruebas para medir variables, que se han considerado actitudes: las variables independientes se le aplicaron al grupo experimental, y las variables dependientes al grupo control. Obtenidos los resultados, se analizaron, y se verificó si la aplicación de las variables independientes, eran la responsable de la mejora en los resultados de la evaluación.

La experiencia educativa abarcó el período escolar 2006/2007 y la llevamos a cabo como ya hemos dicho en el I.E.S. Universidad Laboral de Málaga.

Se han elegido actitudes que los alumnos/as manifiestan como conductas en las clases de Educación Física. Si se logra que las conductas negativas desaparezcan o disminuyan se mejorará el proceso de enseñanza aprendizaje del alumnado, al igual que, si se logra que las conductas positivas se manifiesten frecuentemente.

A continuación mostramos las variables dependientes e independientes que se han aplicado al grupo control y al experimental, y las claves de identificación.

\section{Los contenidos}

Los contenidos se han desarrollado en dos sesiones de trabajo por semana y curso, de aproximadamente $45^{\prime}$. En las sesiones se han desarrollado contenidos teóricos y prácticos. Se han dispuesto de 61 sesiones de actividad. Se ha trabajado individual y colectivamente, en función del contenido a trabajar.

Los espacios se han utilizado las pistas polideportivas, la pista de atletismo, otras en todo el espacio del Instituto de Enseñanza Secundaria «Universidad Laboral» y de la Residencia.

El material utilizado, ha sido el adecuado en todo momento y ha estado en función, de las necesidades específicas del trabajo.

Algunas funciones desempeñada por profesores/as:

- Observador de la experiencia

- Analista de los datos recogidos

- Diseñador de las estrategias teórico-práctico

- Animadordinamizador de los aprendizajes

- Corrector de resultados

- Supervisor de las ejecuciones

- Coordinador del proceso

\section{Fundamentación teórica}

La fundamentación teórica finaliza, al cuestionarse el ¿para qué se evalúa? Se evalúa para disponer de informaciones referidas a aspectos de la Educación Física. Se evalúa por compromiso moral y ético. Se evalúa para cumplir con las exigencias que la Ley establece respecto a los procesos educativos. La L.O.G.S.E. establece que la evaluación tiene que tener las siguientes características: ha de ser inicial, criterial, continua, y final.Aconseja la participación del alumnado en los procesos de evaluación, la observación directa y la recogida sistemática de información relevante.

\section{Conclusiones}

Analizados los resultados, se ha llegado a las siguientes conclusiones:

$1^{\text {a }}$-Las alumnas en general presentan mayores dificultades que sus compañeros en la adquisición de procedimientos relacionados con las habilidades motrices.

$2^{\text {a }}$-Se ha observado que en las prácticas en las que se pasaban los test de rendimiento físico, los alumnos abordaron el trabajo como un reto personal, lo que trajo consigo que dichas pruebas fueran una actividad estimulante.

$3^{\mathrm{a}}$--A las chicas pasar los test físicos, les ha supuesto una tarea poco gratificante, pues ha requerido mucho esfuerzo, no visto recompensado porque los resultados obtenidos, ya que en un primer momento, éstos no han sido muy satisfactorios. Perciben que su esfuerzo no está en consonancia con los resultados obtenidos en los tests físicos. Ellas esperaban mejores calificaciones, lo que le ha desencadenado un cierto desencanto.

$4^{\mathrm{a}}$.-El paso de los tests físicos ha supuesto constatar las diferencias que existen entre ambos sexos, en cuanto al rendimiento físico se refiere, lo que a veces ha sido un acicate para trabajar en la línea de equiparar desigualdades, y constatar un hecho relevante, como es la desigualdad de los sexos. 


\begin{tabular}{|c|c|c|}
\hline VARIABLES INDEPENDIENTES & & \\
\hline $\begin{array}{l}\text { EVALUACIÓN EXPERIMENTAL } \\
\text { GRUPO EXPERIMENTAL }\end{array}$ & & \\
\hline ÁREA PROCEDIMENTAL & & \\
\hline \multirow[t]{6}{*}{ 1.Test de la Condición Física (TCF) } & \multicolumn{2}{|l|}{$\begin{array}{l}\text { T. Resistencia Aeróbica-anaeróbica (R) } \\
\text { T. Velocidad de Reacción (VR) } \\
\text { T. Velocidad de Desplazamiento (VD) } \\
\text { T. Elasticidad-Flexibilidad (EF) } \\
\text { T. Coordinación-Potencia (CP) } \\
\text { T. Fuerza-Destreza (FD) } \\
\end{array}$} \\
\hline & Test de Fuerza y Destreza (FD) & $\begin{array}{l}\text { Fuerza y Destreza (FD1) } \\
\text { Fuerza y Destreza (FD2) } \\
\text { Fuerza y Destreza (FD3) }\end{array}$ \\
\hline & Test de Coordinación Potencia (CP) & $\begin{array}{l}\text { Coordinación Potencia (CP1) } \\
\text { Coordinación Potencia (CP2) } \\
\text { Coordinación Potencia (CP3) }\end{array}$ \\
\hline & Test de Potencia & $\begin{array}{l}\text { Potencia (P1) } \\
\text { Potencia (P2) } \\
\text { Potencia (P3) }\end{array}$ \\
\hline & Test de Equilibrio & $\begin{array}{l}\text { Equilibrio (E1) } \\
\text { Equilibrio (E2) } \\
\text { Equilibrio (E3) }\end{array}$ \\
\hline & Test de Relajación & $\begin{array}{l}\text { Relajación }(\mathrm{Rj} 1) \\
\text { Relajación }(\mathrm{Rj} 2)\end{array}$ \\
\hline $\begin{array}{l}\text { 2.Test de Habilidades y Destrezas Deportivas } \\
\text { (THDD) }\end{array}$ & De Voleibol & \\
\hline $\begin{array}{l}\text { 3.Test de Habilidades y Destrezas Deportivas } \\
\text { (THDD) }\end{array}$ & De salto de Altura & \\
\hline $\begin{array}{l}\text { 4.Test de Habilidades y Destrezas Deportivas } \\
\text { (THDD) }\end{array}$ & De Orientación & \\
\hline $\begin{array}{l}\text { 5.Test de Habilidades y Destrezas Deportivas } \\
\text { (THDD) }\end{array}$ & De Vallas & \\
\hline ÁREA CONCEPTUAL & $\begin{array}{l}\text { 1.Faltas sin justificar (F) } \\
\text { 2.Faltas justificadas (FJ) } \\
\text { 3. Retrasos a clase sin justificar (R) } \\
\text { 4.Retrasos a clase justificados (RJ) } \\
\text { 5.Actitudes positivas en clase (+) } \\
\text { 6.Actitudes negativas en clase (-) } \\
\text { 7.Asistencias sin trabajar y sin justificar (Antnj) } \\
\text { 8.Asistencias sin trabajar justificadas (Antj) }\end{array}$ & \\
\hline 1.Examen Teórico Trimestral (EXA) & $\begin{array}{l}\text { 1.Socialmente (EAS) } \\
\text { 2.Procedimentalmente (EAP) } \\
\text { 3.Conceptualmente (EAO) } \\
\text { 4.Actitudinalmente (EAA) }\end{array}$ & \\
\hline ÁREA ACTITUDINAL & & \\
\hline 1.Registros Conductuales & & \\
\hline 2.Encuestas por Ámbitos de Actuación & & \\
\hline
\end{tabular}

$5^{\mathrm{a}}$.-Los alumnos en general, y especialmente las alumnas, cuando comprueban que se da una cierta relación entre trabajo, y mejora de las calificaciones de tests de condición física, aceptan como necesaria el esfuerzo, si se quiere mejorar el rendimiento físico.

$6^{\text {a }}$-El paso de los tests de habilidades y destrezas deportivas en las chicas, ha provocado desilusión, ya que han visto diferencias técnicas a favor de sus compañeros. Esto en algunos casos ha supuesto un acicate de superación, en otros casos no.

$7^{\mathrm{a}}$.-En general las alumnas, obtienen mejores calificaciones que los chicos en le ámbito conceptual, porque asimilan mejor y estudian más los contenidos conceptuales.

$8^{\mathrm{a}}$-Las chicas pronto se dan cuanta que es más fácil superar la materia, obteniendo buenas calificaciones en el área conceptual, que los chicos, que estudian menos y confían más en sus posibilidades físicas como forma de superar la materia.

$9^{\mathrm{a}}$.-Las chicas perciben sus carencias en cuanto al rendimiento físico, pero pronto se dan cuenta también que superando los aspectos teóricos de la materia, es una estrategia para superar la materia, y obte- ner buenas calificaciones lo que equipara diferencias con sus compañeros. Por ejemplo, ellas obtienen mejores calificaciones en trabajos de dominio artístico que los chicos.

$10^{\mathrm{a}}$.-Este diseño metodológico de evaluación que tiene presente el área de los conceptos, favorece las expectativas de las chicas, que la utilizan como estrategia de aprendizaje de la teoría.

$11^{\mathrm{a}}$.-El sistema de evaluación experimental no perjudica a ningún sexo, más bien todo lo contrario, da igualdad de oportunidades y equipara, a unos y a otros.

$12^{\mathrm{a}}$.-La inclusión de las actitudes en la evaluación ha sido un acierto, puesto que ha reportado buenos resultados a todas las calificaciones, y además, ha contribuido a consolidar actitudes positivas y a mitigar actitudes consideradas negativas.

$13^{\text {a }}$-La calificación del profesor es la resultante de las observaciones, de los registros, de los análisis, y de las reflexiones que ha llevado parejo el proceso.

$14^{\mathrm{a}}$.-Las chicas obtienen mejores calificaciones en el área de las actitudes, porque su comportamiento y actitud es mejor que la de los 


\begin{tabular}{|c|c|}
\hline \multicolumn{2}{|r|}{ RAZONES DEL PORQUÉ SE EVALÚA } \\
\hline INFORMAR & $\begin{array}{l}\text {-A los padres y madres. } \\
\text {-A los alumnos. } \\
\text {-Al personal interesado. } \\
\text {-A los profesores. } \\
\text {-Sobre niveles de partida. } \\
\text {-Sobre posible niveles de llegada. } \\
\text {-Sobre determinados aspectos de la Educación Física. } \\
\text {-Sobre los procesos de evaluación. }\end{array}$ \\
\hline CUMPLIMENTAR & -Exigencias que marca la ley. \\
\hline ORIENTAR & $\begin{array}{l}\text {-Terapéuticamente. } \\
\text {-A talentos deportivos. } \\
\text {-Salidas profesionales. } \\
\text {-Físico-deportivamente. } \\
\text {-Adquisición de hábitos higiénicos saludables. }\end{array}$ \\
\hline PREDECIR & $\begin{array}{l}\text {-Deficiencias, anomalías y diversas patologías psicológicas y físicas. } \\
\text {-Talentos deportivos. }\end{array}$ \\
\hline REFLEXIONAR SOBRE & $\begin{array}{l}\text {-La evolución del proceso de enseñanza-aprendizaje. } \\
\text {-Evolución del proceso metodológico. } \\
\text {-Grado de cumplimiento del Programa Educativo. } \\
\text {-Grado de cumplimiento de los Objetivos Generales, del Ciclo, del Área, y Específicos } \\
\text {-El perfeccionamiento y formación del profesorado. } \\
\text {-Grado de cumplimiento de los objetivos específicos de cada unidad didáctica. } \\
\text {-Evolución sobre Evaluación en la Educación Física. }\end{array}$ \\
\hline COMPARAR & -Las finalidades que se pretenden alcanzar y las logradas. \\
\hline SABER & -Lo que piensan y sienten de aspectos concretos relacionados con la Educación Física. \\
\hline PARTICIPAR & -Directamente en los procesos de evaluación. \\
\hline VALORAR & $\begin{array}{l}\text {-Disponer de datos e informaciones. } \\
\text {-Recoger, almacenar, analizar, procesar informaciones y tomar decisiones. } \\
\text {-Datos del ámbito social. } \\
\text {-Datos del ámbito cognitivo. } \\
\text {-Datos del ámbito afectivo. } \\
\text {-Datos del ámbito motriz. } \\
\text {-Que los alumnos/as participen en los procesos de evaluación } \\
\text {-Disponer de instrumentos de recogida, almacenamiento, interpretación y procesamiento } \\
\text { de la información, lo que va a facilitar la toma de decisiones. } \\
\text {-Construir argumentaciones "cuasi" objetivas a la hora de calificar. } \\
\text {-Manejar estrategias cognitivas para disminuir los niveles de estrés y ansiedad que provoca } \\
\text { los procesos de evaluación y calificación en profesores/as y alumnos/as } \\
\text {-La opinión de los alumnos/as. }\end{array}$ \\
\hline EVALUAR & $\begin{array}{l}\text {-A los alumnos/as } \\
\text {-Al profesor/a } \\
\text {-Procesos metodológicos: objetivos, contenidos, evaluación, instalaciones y material. }\end{array}$ \\
\hline CALIFICAR & -A los alumnos/as. \\
\hline
\end{tabular}

chicos, pero también, porque perciben antes que los chicos, la relación que existe entre una buena actitud y una buena calificación.

$15^{\mathrm{a}}$.-El registro de actitudes es un buen instrumento de, recogida de datos relacionados con el ámbito del conocimiento actitudinal, lo que facilitará la objetividad y el posterior análisis de las conductas objeto de observación y registro.

$16^{\mathrm{a}}$.-La importancia del registro de actitudes, vendrá determinado por el número y por las actitudes objeto de estudio.

$17^{\mathrm{a}}$.-Cuantas más conductas sean observadas y registradas, más información se dispondrá sobre este área, lo que hará disminuir las probabilidades de errar en el diagnóstico, sin embargo, el proceso será más complejo.

$18^{\mathrm{a}}$.-La encuesta por ámbitos de actuación es un instrumento que facilita: La participación del alumnado, lo que le permite que sea partícipe de su evaluación. De su utilización se derivan buenas calificaciones. Es un reforzador de conductas positivas pues las calificaciones que se obtienen son positivas.

$19^{\mathrm{a}}$.-Los alumnos y las alumnas obtienen calificaciones similares en la encuesta por ámbitos de actuación.

$20^{\mathrm{a}}$.-La utilización de este modelo permite: Dar informaciones de diversa índole. Fijar posicionamientos, una vez que se ha adjudicado una calificación. Dar explicaciones a alumnos, padres, profesores, etc. Argumentar las calificaciones, por qué estas y no otras. Entrenar las capacidades de diseño, registro, análisis, observación e interpretación de datos.

$21^{\text {a }}$.-Utilizar una metodología similar requiere, altas dosis de humildad, paciencia, ganas de trabajar, y sobre todo un profundo compromiso educativo.

Con los resultados académicos obtenidos por el grupo Experimental han mejorado en calidad y en cantidad, a los obtenidos por el grupo Control, el Departamento de Educación Física del I.E.S. Universidad Laboral de Málaga, decide adoptar el sistema de evaluación propuesto.

\section{Lecturas}

Para finalizar este artículo, se recomienda la lectura de las siguientes referencias bibliográficas al objeto de poder tener una visión más completa sobre los aspectos de la evaluación en Educación Física:

De la revista Apunts el no 62 (2000): «Buscando una evaluación formativa en educación física: Análisis crítico de la realidad existente, presentación de una propuesta y análisis general de su puesta en práctica» de Víctor Manuel López Pastor.

En este documento se realiza un breve repaso a la temática de la evaluación en $\mathrm{EF}$, como paso previo para realizar una propuesta y presentar algunos de los resultados más generales de su puesta en 
práctica por un grupo numeroso de profesores de EF a lo largo de sus años de experiencia.

El trabajo comienza explicando la evolución del grupo de trabajo, y las razones que les han llevado a esforzarse por buscar sistemas e instrumentos de evaluación en $\mathrm{EF}$ más formativos.

En el segundo apartado, se realizan una serie de reflexiones sobre la temática de la evaluación, relacionándola con marcos de pensamiento y racionalidad más amplios, que ayudan a comprenderla mejor.

En el tercero, se desarrolla una serie de críticas y reflexiones sobre los modelos de evaluación dominantes en EF.

En el cuarto apartado se realiza una propuesta sobre posibles líneas de trabajo y actuación a la hora de buscar una evaluación formativa en EF, y se presenta un breve análisis de los resultados más generales de los sistemas e instrumentos de evaluación contrastados en la práctica.

De la revista Apunts el n 04 (2006): «Análisis de los instrumentos de observación empleados para el registro de variables temporales en educación física» de Luis Lozano Moreno, Jesús Viciana Ramírez y Maurice Piéron.

Se trata de un artículo descriptivo cuyo objetivo fundamental es realizar una recopilación de los distintos instrumentos relacionados con el registro de variables temporales utilizados en la observación de los comportamientos de los alumnos, por un lado, y de los profesores por otro, dentro de las clases de Educación Física (EF).

Los instrumentos y técnicas de observación están agrupados en función del elemento fundamental de estudio, obteniendo tres clasificaciones: a) los relacionados con la observación del comportamiento del alumno; b) con la observación del comportamiento del profesor, c) instrumentos globales. De cada instrumento se destaca su uso más importante, estudios donde ha sido aplicado, así como las variables y/o categorías de análisis.

De la revista Apunts el no 86 (2006): «Resultados de un caso práctico de autoevaluación en educación física en la etapa de primaria» de Nuria Ureña Martín, Fernando Ureña Villanueva, Antonio P. Velandrino Nicolás, FranciscoAlarcón López.

En este artículo se muestran los resultados de la puesta en práctica de un instrumento de Autoevaluación en la Etapa de Primaria con un grupo concreto de alumnos/as.

La finalidad de este sistema de Autoevaluación es permitir la participación de los mismos en su proceso de enseñanza y aprendizaje para el contenido de la habilidad básica de manejo de móviles (lanzamientos, recepciones y botes).

Los resultados se obtuvieron a partir de cuestionarios de Autoevaluación diseñados y preparados para cada uno de los ciclos que conforman dicha Etapa (Ureña, 2004).

Así mismo se cree indispensable las consultas y las lecturas de los siguientes artículos aparecidos en la revista de Educación FísicaApunts: №31. Marzo 1993. Dossier:

«Evaluación en la Actividad Física y el Deporte. Perspectivas de la Evaluación en Educción Física y Deporte» Domingo Blázquez Sánchez. «LaEvaluación de la Educación en la Reforma Educativa» Enric M. Sebastián Obrador.

«LaEvaluación de las Estrategias en Educación Física» V. Navarro y G. Fernández.

«La Evaluación de la Educación Física en el tercer nivel de concreción de la Reforma Educativa» J. Díaz.

«Educación para la salud en la escuela. Aspectos a evaluar desde la Educación Física» M. Corbella.

«Evaluación de programas: un programa de Educación Física y Salud» J. Devis y C. Peiró.

Apunts: №34. Diciembre 1993.

«Aspectos críticos y cívicos del rol de los/las profesionales de la Educación Física y el Deporte: conexiones con la política, la economía y el medio ambiente» Juan Miguel Fernández-Balboa.

«La credibilidad de la investigación cualitativa en al enseñanza de la Educación Física» F. Villar.

\section{Revistas Digitales}

Revistas digitales en la que se pueden efectuar consultas de interés y en la que pueden aparecen artículos relacionados con la presente investigación recomendamos, las que siguen a continuación:

1. WWW.efdeportes.com

2. WWW.wanceulen.com

3. WWW.deportedigital.galeon.com

4. WWW.rendimientodeportivo.com

5. WWW.maestroteca.com

6. WWW.inderef.com

7. WWW.oei.es

8. WWW.educasites.net

Consultar también la Tesis Doctoral «Programa de Evaluación de las Actitudes en Educación Física para la Educación Secundaria Obligatoria» de Manuel Francisco Escribano Castillo y dirigida por los doctores José Luis Chinchilla Minguet y José Carlos Fernández García. Universidad de Málaga 2005.

\section{Bibliografía recomendada}

Bissonette, R. (2000). Tareas de Evaluación en la Educación Física en enseñanza primaria. Barcelona: Paidotribo.

Blázquez, D. (1990). Evaluar en educación Física. Barcelona: INDE.

Devís, J. y Peiró, C (1995): Evaluación de programas: un programa de educación fisica y salud. Apunts, 31: 62-69.

Díaz, J. (1993). La evaluación de laEducación Física en el tercernivel de concreción de la Reforma educativa. Apunts, 31.

Díaz Lucea, J. (1994). La evaluación como un proceso sistemático. Barcelona: INDE.

George, J. D; Fisher,A.; Garth;V.; Pat, R. (2001). Testy pruebas fisicas. Barcelona: Paidotribo.

Heyward, Vivian, H. (2001). Evaluación y prescripción del ejercicio. Barcelona: Paidotribo.

Escribano Castillo, M. F. (2002). Hojas personales de registros de Actitudes porámbitos de actuación. Málaga.

Escribano Castillo, M. F. (2002). Libro de los Cuadernos de Registros. Málaga.

López Pastor, V. (1999): Prácticas de evaluación en educación fisica: estudio de casos primaria, secundaria y formación del profesora$d o$. Valladolid: Universidad de Valladolid.

Martínez López, E. J. (2002). Pruebas de aptitud fisica. Barcelona: Paidotribo.

Martínez López, E. J. (2001). Evaluación informatizada en la Educación Física. Barcelona: Paidotribo.

Salas Blasco, J. (1997). La evaluación de la Educación Física en el tercer ciclo. Una propuesta práctica. Barcelona: INDE.

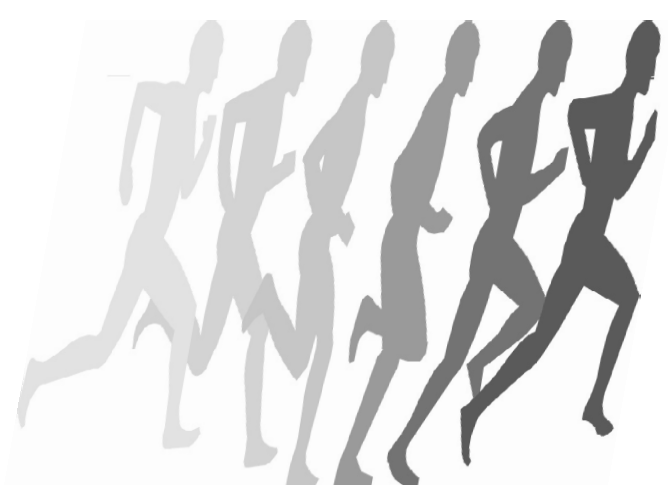

